Revista de Psicología Vol. 36 (2), 2018 (ISSN 0254-9247)

\title{
Análisis psicométrico del Cuestionario de Agresores/Víctimas de Olweus en español
}

\author{
Santiago Resett ${ }^{1}$ \\ Universidad Argentina de la Empresa, CONICET
}

Esta investigación exploró las propiedades psicométricas del Cuestionario Revisado de Agresores/Víctimas de Olweus para adolescentes en español. Se constituyó una muestra intencional de 824 y 1756 adolescentes de la Argentina. Los participantes respondieron al Cuestionario Revisado de Agresores/Víctimas de Olweus, así como a cuestionarios que miden nominaciones de pares, cyberbullying y problemas emocionales y externalizantes. Un análisis factorial exploratorio, como confirmatorio, indicó dos dimensiones bifactoriales relacionadas: una subescala de victimización y otra de agresión; las alfas de Cronbach para ambas subescalas eran adecuadas. Se observó validez convergente de las dos subescalas con los problemas emocionales y externalizantes, como validez de criterio con las nominaciones de pares y confiabilidad test/re-test. Por lo tanto, los resultados de la presente investigación sugieren que el cuestionario de Olweus mantendría una buena bondad psicométrica en la Argentina.

Palabras clave: bullying, victimización, propiedades, validez, confiabilidad

\section{Psychometric Analysis of Olweus Bully/Bullied Questionnaire in Spanish}

The purpose of this study was to explore the psychometric properties of the Revised Olweus Bully/Victim Questionnaire for adolescents in Spanish. A convenience sample of 824 and 1756 high school students in Argentina completed the Revised Olweus Bully/Victim Questionnaire, as well as questionnaires measuring peer nominations, cyberbullying, and internalizing and externalizing problems. Exploratory and confirmatory factorial analysis indicated two related dimensions: one related to being bullied and another related to being and aggressor. Cronbach's Alphas were good. Regarding validity, both subscales suggested construct validity (significant associations with internalizing and externalizing problems) and criterion validity (associations with peer nomination). Also, test re-test reliability was detected. Therefore, results suggest that the Olweus questionnaire has good psychometric properties in Argentina.

Keywords: bullying, victimization, psychometric analysis, validity, reliability

1 Doctor en Psicología. Docente e investigador de la Universidad Argentina de la Empresa. Dirección postal: Paraná, Entre Ríos, Argentina, CP 3100. Contacto: santiago_resett@hotmail.com 


\begin{abstract}
Análise psicométrica do Questionário de Agressores / Vítimas de Olweus em espanhol O objetivo desta pesquisa foi explorar as propriedades psicométricas da versão espanhola do Questionário revisado de Agressores/Vítimas de Olweus para adolescentes. Para este fim, o questionário foi administrado a uma amostra de conveniência de 824 e 1756 alunos das escolas secundárias na Argentina. Os participantes completaram o Questionário revisado de Agressores/Vítimas de Olweus, bem como questionários que medem nomeaçóes de pares, cyberbullying e problemas emocionais e externalizantes. As análises fatoriais indicaram duas dimensôes relacionadas: uma sub-escala de vitimização e outra de agressão. Os Alphas de Cronbach para as duas sub-escalas foram bons. Enquanto a validade do questionário, observa-se validade convergente das sub-escalas com os problemas emocionais e a externalização, e observa-se validade de critério com as nomeaçóes de pares e confiabilidade teste/re-teste. Portanto, os resultados sugeriram que o questionário de Olweus poderia ser uma medida com boas propriedades psicométricas na Argentina.

Palavras-chave: bullying; vitimização; análise psicométrica; validade; confiabilidade
\end{abstract}

\title{
Analyse psychométrique du questionnaire des agresseurs / victimes d'Olweus en espagnol
}

Cette recherche a exploré les propriétés psychométriques du Questionnaire révisé des agresseurs / victimes d'Olweus pour les adolescents en espagnol. Un échantillon intentionnel de 824 et 1756 adolescents d'Argentine a été constitué. Les participants ont répondu au questionnaire d'Olweus ainsi que des questionnaires qui mesurent les nominations par les pairs, la cyberintimidation (ciberbullying) et les problèmes émotionnels et d'extériorisation. Les analyses factorielles, à la fois exploratoires et confirmatoires, ont indiqué deux dimensions bifactorielles connexes: une sous-échelle de victimisation et une autre d'agression; Les alphas de Cronbach étaient adéquats. La validité convergente des deux sous-échelles a été observée avec des problèmes émotionnels et d'externalisation, tels que la validité du critère avec les nominations par les pairs et la fiabilité du test / re-test. Les résultats suggèrent que le questionnaire maintiendrait une bonne qualité psychométrique en Argentine.

Mots-clés: bullying, vitimisation, propriétés, validité, fiabilité. 
El acoso escolar por parte de los pares — bullying en inglés — es un importante factor de riesgo para el ajuste psicosocial de niños y adolescentes (Card \& Hodges, 2008; Swearer \& Hymel, 2015). Existe acoso cuando, en primer lugar, un individuo es expuesto repetidamente a acciones negativas intencionales por parte de un sujeto o grupo y existe una desigualdad de fuerzas entre la víctima y el agresor (Olweus, 1993, 2013). El bullying puede ser llevado a cabo de distintas formas: verbales (poner apodos, burlas, insultos, etc.), físicas (golpes, patadas, empujones, etc.) e indirectamente, esto es, sin usar contacto físico o verbal directo con la víctima (Hymel \& Swearer, 2015; Smith, 2016): esparcir rumores o excluir. En la actualidad el avance de la tecnología y los medios de comunicación, como su popularidad entre los más jóvenes, dio lugar al cyberbullying o acoso electrónico (Olweus, 2012; Kowalski, Giumetti, Schroeder \& Lattanner, 2014; Kowalsky \& Limber, 2013).

Tanto el ser víctima del acoso como el perpetrarlo son factores de riesgo para la psicopatología del desarrollo (Nansel, Craig, Overbeck, Saluja \& Ruan, 2004; Olweus, 2013). Los que son víctimas presentan mayores niveles de problemas emocionales: mayor ansiedad, depresión, peor autoestima y tendencias suicidas (Roh et al., 2015), mientras que los agresores muestran un patrón de problemas de conducta: conducta antisocial, consumo de sustancias tóxicas y potencial conflicto con ley (Farrington \& Ttofi, 2011). Un metaanálisis reciente a partir de 18 estudios longitudinales con adolescentes detectó la asociación entre la victimización con los problemas emocionales, de conductas y los interpersonales, mientras halló la asociación entre el bullying y los problemas externalizantes, los interpersonales y un pobre rendimiento escolar (Klajakovic \& Hunt, 2016).

Los efectos negativos del bullying sobre el desarrollo psicosocial no son solo contemporáneos, sino que pueden persistir en la adultez. Por ejemplo, quienes han sido objeto de este acoso en la escuela media 
pueden experimentar otros problemas emocionales en la adultez - como baja autoestima y más trastornos mentales - que aquellos que no fueron victimizados, como una mayor probabilidad de ser acosados en el trabajo (Copeland, Wolke, Angold \& Costello, 2013). Del mismo modo, los agresores tienen mayor probabilidad de tener problemas con la ley cuando adultos, siendo el bullying uno de los mejores predictores de la conducta criminal futura (Olweus, 2011).

Si bien el bullying puede ser sufrido a lo largo de todo el ciclo vital, en la adolescencia los cambios cognitivos, emocionales y físicos — como las transiciones contextuales académicas y contextuales - aumentan su prevalencia y gravedad (Troop-Gordon, 2017). Las razones de ello son la mayor conciencia del sí mismo, la relevancia psicosocial que cobra el grupo de pares y que los adolescentes pasan mayor tiempo sin supervisión adulta (LaFontana \& Cillesen, 2010), lo cual también limita la eficacia de las intervenciones para disminuir el bullying en dicha etapa (Yearger, Fong, Lee \& Espelage, 2015). Por otra parte, si el acoso se basa en la desigualdad de poder, algunos investigadores afirman que su aumento en los primeros años de la escolaridad media responde también a un intento de establecer dominancia en un contexto nuevo, en el cual los alumnos de menor edad interactúan con pares mayores y el grupo de pares se vuelve más importante, con una relación docentealumno menos personalizada (Pellegrini \& Long, 2010). De este modo, el bullying es más frecuente en la temprana adolescencia, donde la búsqueda de estatus es importante (LaFontana \& Cillesen, 2010).

Numerosos estudios trasnacionales sobre la incidencia del acoso escolar demostraron lo extendido que estaba en distintos países (ej. Cook, Williams, Guerra \& Kim, 2009). Con respecto a su incidencia en poblaciones adolescentes, los estudios indicaron que el bullying era un grave problema. Por ejemplo, un estudio en 40 países detectó que un $26 \%$ de los adolescentes estaba involucrado como víctima o agresor con cierta regularidad (Craig et al., 2009), mientras otros estudios encontraron porcentajes entre 10\%-30\% (Kaltiala-Heino \& Fröjd, 2011).

Debido a que, tanto las víctimas como los agresores, tienen un mayor riesgo de presentar un peor ajuste psicosocial, es vital desarrollar 
y adaptar instrumentos con sólidas bondades psicométricas para su evaluación. Existen numerosas técnicas para medir este problema: observaciones estructuradas, entrevistas, nominaciones de docentes y alumnos, listas de chequeos y autoinformes (Avilés, 2006; Hartung, Little, Allen \& Page, 2011). Cada una de las cuales tiene sus ventajas como desventajas. Los autoinformes, como los cuestionarios, presentan la ventaja de que son técnicas de fácil aplicación e interpretación, con bajos costos económicos y pueden aplicarse en múltiples ocasiones para ver cómo evoluciona el constructo.

Olweus (1996) diseñó el Cuestionario Revisado de Agresores/Víctimas de Olweus (The Revised Olweus Bully/Bullied Questionnaire), el más usado en el mundo para medir el bullying (Cornell \& Bandyopadhyay, 2010). Existen otros autoinformes a este respecto en inglés (Illinois Bully Scale de Espelage \& Holt, 2001; The Bullying Prevalence Questionnaire de Rigby \& Slee, 1993 o Victimization-Bully Scale de Reynolds, 2003), como en espańol o adaptados a dicho idioma (Escala de actitudes ante la agresión social de Villa, 2005; Cuestionario sobre Intimidación y Maltrato entre Iguales de Avilés \& Monjas, 2005 o Escala de Agresión entre Pares de Cajigas, Khan, Luzardo, Najson \& Zalmavide, 2004). Sin embargo, las ventajas de dicho cuestionario son brindar a los alumnos una definición sobre qué se va a entender por acoso, inquirir sobre la victimización y la agresión, preguntar sobre el acoso ocurrido en los últimos meses, presentar una frecuencia temporal para que los alumnos respondan (de "nunca" a "varias veces por semana") e incluir preguntas sobre la actitud de la comunidad educativa (Olweus, 1993, 2013).

Las dos principales dimensiones que mide son ser victimizado y agredir (Kyriakides, Kaloyirou \& Lindsay, 2006). Las investigaciones extranjeras indicaron que presenta una adecuada confiabilidad y validez (Olweus, 2013), las cuales tienden a mantenerse en las distintas naciones. Pese a ser ampliamente usado en el mundo, incluso en los países del primer mundo, no existen muchos estudios publicados sobre sus propiedades psicométricas (Cornell \& Bandyopadhyay, 2010; Kyriakides et al., 2006). Solo dos estudios a este respecto se encontraron en la literatura científica: Kyriakides et al. (2006) en población chipriota griega 
y Hartung et al. (2011) en los Estados Unidos. A pesar de ser los únicos estudios disponibles, se detectó la misma estructura factorial postulada por el autor y adecuadas consistencias internas. En idioma castellano el presente estudio no detectó investigaciones de sus propiedades.

A diferencia del sólido cuerpo de investigación sobre el bullying que existe en los países del primer mundo, en los países latinoamericanos es mucho lo que falta por investigar (Romera Félix, del Rey Alamillo \& Ortega Ruiz, 2011). En la Argentina, existen pocos datos científico-empíricos a este respecto y, menos aún, con instrumentos de reconocidas propiedades. Entre las pocas investigaciones a este respecto, el Observatorio Argentino de Violencia en las Escuelas (2008) halló con muestras representativas de adolescentes una incidencia de más de 30\% de acoso físico y un 15\% de bullying verbal; también el Fondo para la Infancia de las Naciones Unidas (Unicef, 2011) detectó que un $18 \%$ de alumnos era victimizado con burlas por su aspecto físico de manera habitual, con la Argentina ostentando uno de los niveles más alto de bullying a nivel latinoamericano.

Asimismo, los casos de bullying denunciados aumentaron un 33\% del año 2015 al 2016 en niños y adolescentes argentinos (Bullying sin Fronteras, 2016). Sin embargo, el problema de dichos estudios es que no usaron instrumentos de sólidas propiedades psicométricas para medir el acoso. Del mismo modo, no existen estudios en idioma español que hayan examinado la estructura factorial del cuestionario de Olweus, validez de criterio, convergente y confiabilidad test/re-test. Solo se dispone de una adaptación al portugués en el Brasil, la cual evaluó su estructura y confiabilidad con la teoría de la respuesta al ítem (Gonçalves, Heldt, Peixoto, Rodrigues, Filipetto \& Guimarães, 2016) y un análisis de su consistencia interna en una pequeña muestra de adolescentes argentinos (Resett, 2011).

Así el presente trabajo contribuye a la literatura científica al examinar las propiedades de una adaptación al español del cuestionario de Olweus con una muestra de adolescentes de gran tamańo. Para esto, se observó la validez de criterio de dicho cuestionario a partir de nominaciones de pares y la validez convergente de la escala ser victimizado y la de ser 
agresor a partir de su asociación y con un cuestionario de cyberbullying, con los problemas emocionales (depresión y ansiedad) y externalizantes (conducta antisocial y agresividad), respectivamente. Además, se evaluó la consistencia interna y la confiabilidad test-retest de ambas escalas.

\section{Método}

\section{Participantes}

Primero, se constituyó una muestra de 824 alumnos que cursaban estudios de nivel medio en cuatro escuelas públicas de cuatro ciudades de la provincia de Entre Ríos, Argentina. El 46\% eran varones y el promedio de edad era de 14.9 ańos. Luego se constituyó una muestra de 1756 alumnos que cursaban estudios de nivel medio en cinco escuelas públicas de Paraná, Entre Ríos. El 48\% eran varones y el promedio de edad era de 14.7 años. Los criterios de inclusión eran cursar estudios medios en escuelas públicas, ser varón o mujer, tener entre 12 y 18 años y poseer el consentimiento parental para participar. La primera muestra solo contestó el cuestionario de Olweus para llevar a cabo un análisis factorial exploratorio. La segunda muestra contestó el cuestionario de Olweus, nominaciones de pares, un cuestionario de cyberbullying y medidas de problemas emocionales y externalizantes. La misma se usó para un análisis factorial confirmatorio y para responder a los restantes objetivos del trabajo. Seis meses después se evaluaron $48 \%$ de los alumnos $(n=552)$ de la segunda muestra para ver la confiabilidad test/ re-test del cuestionario de Olweus.

\section{Medición}

Cuestionario Revisado de Agresores/Victimas de Olweus (1996). Consiste en 38 preguntas para medir los problemas con relación a agredir/ser victimizado en niños y adolescentes. En primer lugar, da una definición a los alumnos sobre qué se va a entender por acoso escolar. A continuación, los alumnos son interrogados sobre los distintos tipos de acoso que experimentaron —o que llevaron a cabo- a partir de nueve preguntas 
sobre la frecuencia de las distintas formas de ser victimizado y otras nueve sobre agredir: golpear, sacar o romper cosas, poner sobrenombres, burlas sobre el aspecto físico, burlas sexuales, amenazas, excluir, decir mentiras y agredir con mensajes de textos desde el celular o con Internet. Las preguntas sobre victimización o agredir pueden sumarse o promediarse para confeccionar una subescala (Kyriakides et al., 2006).

Un ejemplo de pregunta del Cuestionario de Olweus es: "Me pusieron sobrenombres feos, me hicieron cargadas pesadas, o se burlaron de mí". El cuestionario emplea las siguientes alternativas de respuesta: - Nunca -Una o dos veces - Dos o tres veces al mes-Más o menos una vez por semana $\mathrm{y}-$ Varias veces por semana. Las respuestas son codificadas como 0 (Nunca) a 4 (Varias veces por semana). En la presente investigación solo se informará sobre las dos subescalas, ya que el resto de las preguntas no forman parte de estas.

Las virtudes psicométricas del instrumento fueron comprobadas (Olweus, 2013). En lo relativo a su estructura factorial en muestras de niños y adolescentes, los pocos estudios disponibles indican con análisis factoriales y teorías de la respuesta al ítem una estructura de ser victimizado y una de agredir (Hartung et al., 2011; Kyriakides et al., 2006). Por ejemplo, el estudio de Hartung et al. (2011) halló que dicha estructura bifactorial explicaba un $48 \%$ de la varianza y todos los ítems cargaban por encima de .55 en sus respectivas dimensiones. Su validez de constructo fue comprobada por su asociación lineal con los problemas emocionales para la subescala de victimización y de conducta para la de agredir, como también su consistencia interna con valores alfas de Cronbach entre .80 y .90 (Olweus, 2013; Solberg \& Olweus, 2003). También, se detectó su validez convergente con la escala BullyVictimization Scales (BVS) de Reynolds (Hartung et al., 2011).

Por otra parte, una investigación con 21 estudios halló validez de criterio con distintas nominaciones de pares, como lo indicaba una asociación $r=.37$ con el instrumento de Olweus. Se adaptó y tradujo en numerosos países y lenguas (Kyriakides et al., 2006). En el presente estudio se empleó el cuestionario de Olweus que Resett (2011) adaptó a la Argentina. La adaptación al espańol argentino fue realizada por cuatro 
traductores de inglés que hicieron la traducción inversa. Luego cuatro investigadores controlaron la fidelidad de la traducción. Dicho cuestionario adaptado a la Argentina solo presenta una mínima diferencia con el original: el ítem de ser victimizado o agredir mediante burlas en lo relativo al color de piel o raza ("I was bullied with mean names or comments about my race or color") se redactó "Fui agredido con sobrenombres o comentarios feos por mi color de piel" y se omitió la raza. Esto se debe a que la amplia mayoría de los jueces expertos consideró que no era ecológicamente válido en dicho contexto. Finalmente se aplicó en una muestra piloto de 80 alumnos de dos escuelas secundarias quienes los completaron y se les sugirió que verbalicen cualquier tipo de duda. En dicho estudio preliminar se hallaron alfas de Cronbach similares a las informadas por Olweus, como lo eran .87 y .79 (Resett, 2011).

Para evaluar la validez de criterio del Cuestionario de Olweus se aplicó:

Nominación por parte del grupo de pares de Juvonen, Graham y Schuster (2003). Esta permite que los pares identifiquen quiénes son agresores y quiénes son víctimas a partir de los siguientes descriptores: "empuja y agarra a las piñas a otros alumnos"; "se burla, hace cargadas pesadas; "pone sobrenombres feos a otros alumnos" y "dice cosas feas y mentiras sobre otros alumnos". Esta nominación demostró buena confiabilidad interna y validez en los estudios del primer mundo (Juvonen et al., 2003). Las alfas de Cronbach en el presente estudio fueron .84 para los ítems de la escala ser agresor y .75 para los de ser víctima, respectivamente. Para considerar que un alumno es agresor o víctima debe puntuar .5 desviaciones típicas por encima de la media.

Para evaluar la validez convergente de las subescalas del cuestionario de Olweus se aplicó: El Cuestionario de Cyberbullying de Calvete, Orue, Estévez, Villardón y Padilla (2010). Se compone de dos escalas diferentes, una para medir cyberagresión y una para medir cybervictimización. La versión del cuestionario consta de 14 preguntas sobre agredir y 14 sobre victimización. Un ejemplo de pregunta es: "Me enviaron mensajes amenazantes o insultantes con la Internet o el celular". El formato de respuesta utilizado para evaluar la frecuencia 
cada comportamiento es 0 (Nunca), 1 ( 1 o 2 veces), 2 ( 3 o 4 veces), o 3 ( 5 o más veces). Sus propiedades están bien establecidas (Calvete et al., 2010; Gámez-Guadix, Villa-George \& Calvete, 2014). En este estudio las alfas de Cronbach fueron .93. y .87, respectivamente.

Para evaluar la validez convergente de la subescala ser victimizado, se aplicaron las siguientes medidas de problemas emocionales:

Inventario de Depresión para Niños de Kovacs (1992). Este cuestionario, uno de los más usados en el mundo, se emplea en niños y adolescentes de 7 a 17 ańos a partir de 27 preguntas. Un ejemplo de ítems es: "Todo el tiempo me siento triste". La autora informa una consistencia interna de alfa de Cronbach que fluctúa entre .71 y .89 para distintas muestras (Kovacs, 1992). El alfa de Cronbach fue .83 en la presente muestra.

Escala Rosenberg de Sintomas Psicosomáticos (1973). Esta escala de 10 preguntas evalúa la ansiedad a través de síntomas de activación del sistema nervioso autónomo. La escala presenta cuatro opciones de respuesta de 0 a 3 (Nunca a Algunas veces o a menudo). Un ejemplo de ítem es: "Me preocupan mis nervios". El alfa de Cronbach fue .84 en la presente muestra.

Para evaluar la validez convergente de la subescala ser agresor, se aplicaron las siguientes medidas de problemas externalizantes:

Escalas de agresividad y conducta antisocial del Autoinforme para Jóvenes de Achenbach y Rescorla (2001). Estas inquieren sobre síntomas de agresividad (17 preguntas) y conducta antisocial (15 preguntas) con tres opciones de respuestas de 0 a 2 (No es cierto a Muy cierto). Un ejemplo de ítem es: "Discuto mucho". La fiabilidad y validez de este instrumento en grupos de adolescentes ha sido ampliamente comprobada en numerosos estudios extranjeros (Achenbach \& Rescorla, 2001). En la presente muestra las alfas de Cronbach fueron .85 y .84, respectivamente.

\section{Procedimiento}

En primer lugar, se contactó a los directores de las escuelas con el fin de solicitar la autorización de ellos y de los padres. Se aseguró a 
los jóvenes la confidencialidad y el anonimato de las respuestas. También se aseguró la participación voluntaria y se pidió el consentimiento informado de los padres o tutores por escrito mediante una nota en el cuaderno de comunicaciones de los alumnos. La medición pre-test del cuestionario de Olweus se aplicó en mayo de 2014 y la pos-test en noviembre de 2014 .

\section{Análisis de datos}

Los datos se analizaron en el programa Estadístico para las Ciencias Sociales SPSS versión 22 y el programa AMOS 16. Para evaluar la estructura factorial se llevaron a cabo análisis factoriales exploratorios y confirmatorios; para determinar la validez convergente y confiabilidad test/re-test, correlaciones de Pearson; y para la confiabilidad interna se calcularon alfas de Cronbach. Se tomó como criterio de significación estadística $p<.05$. Para el ajuste del modelo en el análisis factorial confirmatorio se tuvieron en cuenta el Índice Comparativo de Ajuste (CFI), el Tucker-Lewis Index (TLI) y el promedio de los residuales estandarizados al cuadrado (RMSEA), ya que son los índices de ajuste más usados (Byrne, 2010). Se usó el procedimiento de máxima verosimilitud.

\section{Resultados}

Para evaluar la estructura factorial del Cuestionario de Olweus, se realizó un análisis factorial exploratorio con los nueve ítems de victimización y agresión mediante el método de componentes principales. Se solicitó la extracción de autovalores mayores a 1 y una rotación Oblimin debido a que se hipotetizaba que los factores estarían correlacionados. El cálculo del índice de adecuación muestral Kaiser-Meyer-Oklin $\mathrm{KMO}=.91$ y la prueba de esfericidad de Bartlett $X^{2}=3754.22 ; p<$ .001 indicaron que era apropiado llevarlo a cabo.

En la tabla 1 se presentan los resultados del análisis factorial, el cual arrojaba dos factores que explicaban un $51 \%$ de la variancia. 
El primero se podía denominar victimización y estaba compuesto por los nueve ítems de dicha subescala. El otro se denominó agresión y se conformaba por los ítems de ser agresor. Cada pregunta cargaba en su correspondiente factor y no existían cargas cruzadas mayores a .30. La correlación entre ambas subescalas era $r=.57 p<.001$.

\section{Tabla 1}

Cargas factoriales para las preguntas del Cuestionario de Olweus de victimización y agresión

Ítem

Cargas factoriales

Victimización Agresión

Mentiras

.73

sobrenombres feos

.77

burlas sobre aspecto físico

.73

burlas sexuales

.70

Exclusión

.67

Amenazas

.44

sacar o romper cosas

.56

Golpes

.50

SMS o con Internet (bullying electrónico) .48

Mentiras

sobrenombres feos

burlas sobre aspecto físico

burlas sexuales

Exclusión

Amenazas

sacar o romper cosas

Golpes

SMS o con Internet (bullying electrónico) 
Luego se procedió a llevar a cabo un análisis factorial confirmatorio con la segunda muestra. Para considerar si el modelo era aceptable, se tuvieron en cuenta el Índice Comparativo de Ajuste (CFI), el TuckerLewis Index (TLI) y el promedio de los residuales estandarizados al cuadrado (RMSEA). Se puso a prueba un modelo de dos factores relacionados (victimización y agresión) con las correlaciones entre los errores de medición de las preguntas que inquirían conductas similares, como si fueran medidas repetidas (Modelo 1); ya que estudios previos demostraron que los errores de los ítems de ser victimizado y agredir tienden a estar asociados en instrumentos que miden el mismo constructo (GamezGuadix et al., 2014). Dicho modelo se presenta en la figura 1.

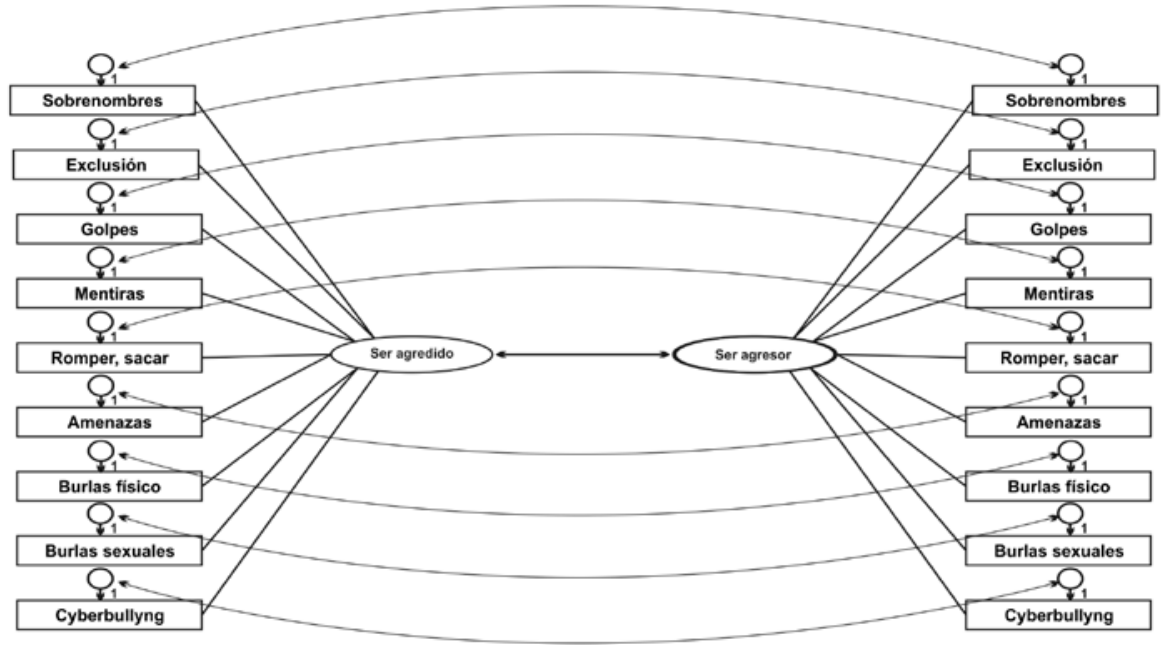

Figura 1. Modelo del Cuestionario de Olweus con victimización y agresión como factores relacionados y con asociaciones entre sus errores de medición.

Los resultados mostraron un ajuste adecuado para el modelo 1, como se ve en tabla 2. El CFI era .95, el TLI, .92 y el RMSEA, .05. Se consideran valores de CFI y TLI por encima de .90 y RMSEA por 
debajo de 10 como adecuados (Bentler, 1992; Byrne, 2010). También los valores estaban sumamente cercanos a criterios más exigentes con más de .95 y menos de .05 , respectivamente (Hu \& Bentler, 1999).

\section{Tabla 2}

Índice de ajuste para el modelo del Cuestionario de Olweus para las preguntas victimización y agresión

\begin{tabular}{cccccc}
\hline Modelo & $\chi^{2}$ & $g l$ & $C F I$ & TLI & RMSEA \\
\hline Dos factores relacionados & 902.03 & 125 & .95 & .92 & .05 \\
\hline
\end{tabular}

Nota. ${ }^{* * *}<p .001 \mathrm{gl}=$ grados de libertad. CFI = Comparative Fixt Index. TLI = Tucker-Lewis Index. RMSEA = residuales estandarizados al cuadrado.

Las cargas factoriales para el modelo iban de .49 a .75 para victimización y de .53 a .71 para agresión. Para ambos factores la asociación era $r=.59 p<.001$. La media para la subescala victimización era 2.74 $(D E=4.22)$ y para agresión era $1.75(D E=1.31)$.

En cuanto a las nominaciones por parte del grupo de pares, un $5 \%$ de los alumnos recibió al menos dos nominaciones que lo señalaba como víctima. Un 15\% recibió al menos dos nominaciones que lo seńalaba como agresor. Al promediar los tres ítems, se obtuvo una media de $.38(D E=.30)$ para ser victimizado y de $.66(D E=.64)$ para ser agresor.

$\mathrm{Al}$ correlacionar las nominaciones de pares y el autoinforme para observar la validez de criterio de ambas subescalas, se halló $r=.32$ ( $p$ $<.01)$ entre los puntajes de las nominaciones para ser victimizado y los puntajes de la subescala de Olweus, y $r=.25(p<.01)$ entre ambos informes para ser agresor.

Para explorar la validez convergente de las subescalas victimización y agresión, se realizaron correlaciones de Pearson entre estas y las subescalas cybervictimización y cyberagresión del cuestionario de Calvete et al. (2010). Con el fin de evaluar la validez convergente de la subescala victimización, se realizó una correlación de Pearson entre esta y los puntajes de depresión y ansiedad. El mismo procedimiento se llevó a 
cabo con la subescala de agredir y los puntajes de conducta antisocial y agresividad. En la tabla 3 se presentan los resultados, los cuales mostraban correlaciones significativas y en la dirección esperada. Para la subescala victimización de Olweus, las asociaciones eran $r s=.38, .32 \mathrm{y}$ .64 ( $p s<.001)$ con depresión, ansiedad y ser cybervictimizado, respectivamente. Para la subescala agresión, eran $r s=.44, .42$ y $.58(p s<.001)$ con conducta antisocial, agresividad y ser cyberagresor,

\section{Tabla 3}

Correlaciones entre las subescalas victimización y agresión de Olweus y las escalas de problemas emocionales, de conducta y las escalas de cybervictimización y ser cyberagresor del Cuestionario de Cyberbullying

\begin{tabular}{lcc}
\hline Escalas & $\begin{array}{c}\text { Subescala victimización } \\
\text { de Olweus }\end{array}$ & $\begin{array}{c}\text { Subescala agresión } \\
\text { de Olweus }\end{array}$ \\
\hline Depresión & $.38^{* * *}$ & - \\
Ansiedad & $.32^{* * *}$ & - \\
Conducta antisocial & - & $.44^{* * *}$ \\
Agresividad & - & $.42^{* * *}$ \\
Escala cybervictimización & $.64^{* * *}$ & - \\
Escala cyberagresor & & $.58^{* * *}$ \\
$n=1756$ & & \\
\hline
\end{tabular}

Nota. ${ }^{* * *} p<.001$

En lo relativo a la consistencia interna, al calcular el estadístico alfa de Cronbach para la primera muestra, el mismo fue .87 y .83 para las subescalas victimización y agresión, respectivamente. Con respecto a la segunda muestra, los valores eran .88 y .82 , respectivamente.

Con en el fin de ver la confiabilidad test/re-test, se llevaron a cabo correlaciones de Pearson. Los resultados de las mediciones test/re-test indicaban asociaciones significativas con $r=.62, p<.01$ para la subescala victimización y $r=.56, p<.01$ para agresión, respectivamente. 


\section{Discusión}

El bullying es un importante factor de riesgo para la salud mental de niños y adolescentes. Así la importancia de esta investigación radicaba en evaluar las propiedades psicométricas del Cuestionario Revisado de Agresores/Víctimas de Olweus (1996) en una muestra intencional de 824 y 1756 alumnos adolescentes de escuelas medias argentinas. A pesar de ser el instrumento más usado en el mundo a este respecto, no existen datos empíricos sobre sus propiedades psicométricas en naciones latinoamericanas de habla española. Incluso la literatura científica sobre sus propiedades es escasa en los países del primer mundo.

En lo referente al análisis factorial exploratorio del instrumento, en la muestra de 824 alumnos emergían dos factores que explicaban un $51 \%$ de la varianza. Los dos factores se llamaron victimización y agresión; el primero se componía de las nueve preguntas de victimización y el segundo, de las nueve de agresión. Se observaron adecuadas cargas factoriales y no existían cargas cruzadas mayores a .30. El análisis factorial confirmatorio en la muestra de 1756 alumnos también arrojó un adecuado modelo de medición $(\mathrm{CFI}=.95$ y TLI $=.92)$ con dos factores relacionados para los nueve ítems de victimización y agresión, respectivamente. Dichos resultados concuerdan con la estructura factorial hallada por Kyriakides et. (2006) en población chipriota griega, Hartung et al. (2011) en una muestra norteamericana y Breivik y Olweus (2015) en muestras noruegas.

En lo referente a la validez de criterio, al relacionar la subescala victimización de Olweus con las nominaciones de pares de Juvonen et al. (2003), la misma fue $r=.32$. La asociación para agresión entre ambas técnicas fue $r=.25$. Revisiones meta-analíticas con 21 estudios (Card, 2003) examinaron las correlaciones entre autoinformes -algunos empleando el instrumento de Olweus- y nominaciones de pares y también hallaron una asociación modesta $(r=.37)$. Entre los pocos estudios recientes que se disponen, Karna, Voeten, Little, Poskiparta, Alanen y Salmivalli (2011) hallaron una asociación de .40 entre la subescala victimización de Olweus y el informe de pares y .34 para ser agresor. 
Aunque las asociaciones entre ambas técnicas son generalmente significativas, como señala Olweus (2013), las mismas generalmente no son elevadas. Existen fuertes razones para ello: el bullying es algo que muchas veces pasa desapercibido y -en ocasiones- solo tiene conocimiento la víctima. Otra cuestión es que muchas veces el acoso es llevado a cabo por pares de cursos o grados diferentes, por lo cual esto no es detectado en las nominaciones. Por otra parte, el cuestionario de Olweus pregunta sobre la frecuencia de ser acosado o agredir. En cambio, las nominaciones de pares buscan que se identifiquen "casos extremos" de alumnos que son victimizados o agresores. Finalmente, el bullying hacia las mujeres es generalmente llevado a cabo por varones. Que las mujeres principalmente sean acosadas por varones y que ellos, a su vez, intimiden en mayor medida, puede ser algo no tan bien captado por las nominaciones de pares, ya que los alumnos tienden a enfocarse en sus pares del mismo sexo.

También ambas subescalas correlacionaron .64 y .58 con un índice de ser cybervictimizado y ser cyberagresor del Cuestionario de Calvete et al. (2010), respectivamente, lo cual demuestra su validez convergente. El primer estudio sobre el cyberbullying se llevó a cabo en el año 2000, por ende, la investigación sobre el mismo es algo limitada en comparación con el bullying tradicional (Raskauskas \& Stoltz, 2007). Numerosas investigaciones sugieren que la popularidad de Internet y las nuevas tecnologías han hecho que surja este nuevo tipo de acoso (Mason, 2008). En la literatura científica de países tecnológicamente más avanzados, para algunos el cyberbullying es simplemente una nueva forma de acoso escolar con las nuevas tecnologías de la información y comunicación (Olweus, 2012; Olweus \& Limber, 2018). En cambio, para otros, es un fenómeno con características distintas, como anonimato, viralización, desinhibición y presencia más allá del ámbito escolar (Mehari, Farrel \& Le, 2014), lo cual lo vuelve un fenómeno cualitativamente diferente (Kowalski \& Limber, 2013; Sontang, Clemans, Graber \& Lyndon, 2011). Como bien señalan Kowalski y Limber (2013), existe una asociación entre ambos constructos, aunque esta no es perfecta por las razones antedichas. Sin embargo, muchos estudios indicaron 
que existe un grado importante de solapamiento entre el bullying y el cyberbullying, ya que muchos de los sujetos que eran victimizados también sufrían de cybervictimización y lo mismo sucedía para la agresión (Raskauskas \& Stoltz, 2007). Patchin y Hinduja (2015) señalan que el cyberbullying es un daño intencional y repetido hacia otra persona mediante computadoras, celulares u otros medios electrónicos y -en ocasiones- con un componente de desigualdad. Dichas características son también las que diferencian al bullying de otros tipos agresión, por lo cual el bullying y el cyberbullying tienen elementos comunes (Patchin \& Hinduja; 2015). En el presente estudio las asociaciones entre la victimización y a la cybervictimización, por un lado, y entre la agresión y la cyberagresión, por el otro lado, fueron satisfactorias (por encima de .50), lo cual es una prueba de validez de constructo del cuestionario de Olweus.

En lo referente a la validez convergente de la subescala victimización, esta se asociaba significativamente con la escala de depresión y la medición de ansiedad ( $r s=.38$ y .32 , respectivamente). En lo relativo a la validez de la subescala agresión, esta se correlacionaba con la escala de conducta antisocial y agresividad ( $r s=.44$ y .42 , respectivamente). Que la subescala victimización correlacione linealmente con los problemas emocionales, como depresión y ansiedad, mientras que la de agresión lo haga con otras conductas externalizantes es algo que también fue detectado en los estudios extranjeros (Olweus, 2013).

Está sólidamente probado que las víctimas del acoso presentan mayores problemas emocionales, principalmente depresión y ansiedad, mientras que los agresores muestran un patrón de problemas externalizantes (Cook, Williams, Guerra \& Kim 2010; Olweus, 2013; Ttofi, Farrington \& Lösel, 2011). La asociación entre dichos problemas emocionales y la subescala victimización puede entenderse por el hecho de que los sujetos ansiosos y depresivos son un blanco más común del acoso. Por ejemplo, la depresión puede contribuir a dificultades con los pares debido a que estos alumnos exhiben un déficit en las habilidades sociales, lo cual puede generar respuestas agresivas por parte de los pares (Rudolph, Flynn \& Abaied, 2008). Pero también por el hecho 
de que las personas depresivas se involucran en relaciones conflictivas (Rudolph, 2009). Sin embargo, se comprobó también que los niveles de problemas emocionales más elevados en las víctimas son una consecuencia del maltrato (Kaltiala-Heino, Frojd \& Marttunen, 2010).

En lo referente a la asociación entre la subescala agresión y los problemas de conducta o externalizantes, es sabido que los agresores presentan niveles más elevados de problemas de conducta antisocial y agresividad que los grupos no agresores, como señalan las revisiones meta-analíticas (Cook et al., 2010; Farrington \& Ttofi, 2011). El que los agresores presenten mayores problemas externalizantes, puede comprenderse desde la teoría de los problemas de conducta de Jessor y Jessor (1977), la cual establece que dichos problemas tienden a coocurrir en algunos sujetos debido a que tienen una personalidad desviada, lo cual explica la asociación aquí detectada. Que la relación entre la subescala ser agresor de Olweus y la de agresividad haya sido mediana con un $r=.42$ no es llamativo, ya que como bien señala Olweus (2013), el bullying es un subtipo de agresión con características especiales, como la repetición y la desigualdad de fuerza mental y/o física. Así estos hallazgos nuevamente son una prueba de la validez convergente de ambas subescalas.

En lo concerniente a los alfas de Cronbach, las mismas fueron muy adecuadas, con valores de 87 y .83 para las subescalas victimización y agresión en la primera muestra y 88 y .82, para la segunda muestra. Kaplan y Saccuzzo (2006) seńalan que valores entre .70 y .80 se consideran como adecuados, por lo cual, los resultados eran muy satisfactorios. Resultados similares arrojaron estudios de distintos países del primer mundo (Hartung et al., 2011; Kyriakides et al., 2006), los cuales hallaron unos coeficientes entre .80-.90. Estudios recientes de Olweus en muestras noruegas de casi 50000 alumnos detectaron valores semejantes (Breivik \& Olweus, 2015; Olweus, 2013).

Con respecto a la confiabilidad test/re-test a seis meses, los resultados indicaron correlaciones significativas para las subescalas victimización y agresión ( $r s=.62$ y .56 , respectivamente). Esto sugeriría que dicho instrumento es confiable, ya que el tamańo de la asociación era grande. 
A pesar de que dichas asociaciones eran significativas y altas, pueden parecer no excesivamente altas para un período de solo medio año. Una posible explicación puede ser que, a pesar de que existe una fuerte continuidad en el ser acosado como en el ser agresor, también la literatura científica ha hallado una discontinuidad. Así los estudios detectaron diferentes trayectorias para el ser victimizado y agredir en la adolescencia (Reijntjes et al., 2013; Sheppard, Giletta \& Prinstein, 2016).

Por otra parte, está bien establecido que los roles de víctimas y agresores no son fijos, sino que puede haber una modificación de los mismos. Por ejemplo, Olweus $(1993,2013)$ señala que el acoso es un fenómeno relacional, por lo cual está muy afectado por el contexto, como las reacciones de los espectadores, las víctimas o el accionar de los docentes y padres. Por ejemplo, un alumno puede ser victimizado a principios de año, pero luego a finales del mismo año puede salir de dicho rol, tal vez logrando una amistad íntima o gracias al apoyo de docentes o padres. Por otra parte, esta investigación evaluó a sujetos con edades fluctuando entre 12 y 18 ańos. Está comprobado que en la adolescencia media y tardía hay una disminución y una menor continuidad del acoso debido a que los adolescentes desarrollan mayores recursos cognitivos, sociales, un sentido más coherente de identidad y las relaciones entre los grupos de pares son más igualitarias (TroopGordon, 2017). Futuros estudios deberían examinar qué factores explican que un alumno deje de ser victimizado.

A pesar de ser la Argentina un país menos desarrollado y de otra tradición cultural que los del primer mundo, como lo son los países del norte de Europa, los Estados Unidos o el Canadá, estos hallazgos sugerirían que en la presente muestra de habla espańola este instrumento también mantendría su bondad psicométrica. Al ser el primer estudio en idioma español que evaluó en profundidad las propiedades del cuestionario de Olweus, no solo brinda vital información psicométrica, sino que también el presente estudio tiene implicancias prácticas. Muchos autores señalan que es poco probable que las autoridades educativas puedan identificar el bullying mediante la observación de las conductas (Phillips \& Cornell, 2012). Los resultados del presente trabajo indica- 
rían que el instrumento de Olweus es una herramienta válida, confiable y de fácil implementación para medir los alumnos victimizados y agresores, como también una herramienta para medir la eficacia de las intervenciones para disminuir el bullying, ya que un primer paso previo a cualquier intervención es medir la gravedad del problema.

Como señala Olweus (2013), para observar la eficacia de las intervenciones los autoinformes son más indicados que las nominaciones de pares, ya que estos evalúan la experiencia subjetiva de ser victimizado, mientras que las nominaciones miden las reputaciones de los alumnos. Por otra parte, el cuestionario de Olweus (2013), al brindar una caracterización del acoso (lugares donde este ocurre o quiénes lo llevan a cabo), como de las actitudes de los miembros de la comunidad educativa ante el bullying, provee datos vitales para comenzar una intervención. Futuros estudios deberían también evaluar cómo se comportan psicométricamente el resto de las preguntas del instrumento.

Este estudio tiene una serie de limitaciones: haber sido llevado a cabo con una muestra intencional de distintas ciudades de la Provincia de Entre Ríos, Argentina, por lo cual los resultados no son generalizables a toda la población de dicho país, a pesar del tamaño grande de la muestra. Por otra parte, la mayoría de los datos han sido recogidos mediante el autoinforme, lo cual infla artificialmente las relaciones entre las variables por la varianza compartida del método de recolección de datos.

Futuros estudios deberían examinar esta problemática en muestras seleccionadas al azar de diversos países de Latinoamérica para tratar de determinar si el instrumento conserva sus propiedades psicométricas y determinar si mantiene su invariancia de medición a través de los países e idiomas (espańol, portugués, entre otros). Si bien la Argentina pertenece a la región, presenta características culturales, sociales, políticas y económicas distintas: su población desciende de espańoles e italianos en su gran mayoría, presenta una notoria inestabilidad política y económica, entre otras. También en la Argentina se debería trabajar con muestras al azar y de distintas regiones de dicho país, como analizar el instrumento desde la teoría de la respuesta al ítem. 


\section{Referencias}

Achenbach, T. M. \& Rescorla, L. A. (2001). Manual for the ASEBA School-Age Forms y Profiles. Burlington, VT: University of Vermont, Research Center for Children, Youth, and Families.

Avilés, J. (2006). Bullying. Intimidación y maltrato entre el alumnado. Bilbao, España: STEE-EILAS.

Bentler, P. M. (1992). On the fit of models to covariances and methodology to the Bulletin. Psychological Bulletin, 112(3), 400-404. https://doi.org/10.1037/0033-2909.112.3.400

Breivik, K. \& Olweus, D. (2015). An item response theory analysis of the Olweus Bullying Scale. Aggressive Behavior, 41, 1-13. https:// doi.org/10.1002/ab.21571

Bullying sin fronteras. (2016). Informe del observatorio para la República Argentina. Buenos Aires: Bullying sin fronteras.

Byrne, B. (2010). Structural equation modeling with AMOS: Basic concepts, applications, and programming. Nueva York, NY: Routledge.

Calvete, E., Orue, I., Estévez, A., Villardón, L. \& Padilla, P. (2010). Cyberbullying in adolescents: Modalities and aggressors' profile. Computers in Human Behavior, 26, 1128-1135. https://doi. org/10.1016/j.chb.2010.03.017

Card, N. A. (2003). Victims of peer aggression: a meta-analytic review. Trabajo presentado en la reunión de la Society for Research in Child Development, Tampa, Estados Unidos.

Card, N. A. \& Hodges, E. V. (2008) Peer victimization among school children: correlations, causes, consequences, and considerations in assessment and intervention. School Psychology Quarterly, 23, 451-461. https://doi.org/10.1037/a0012769

Cook, C. R., Williams, K. R., Guerra, N. \& Kim, T. (2009). Variability in the prevalence of bullying and victimization: A cross-national and methodological analysis. En S. Jimerson, S. Swearer y D. Espelage. (Eds.), The International Handbook of School Bullying (pp. 347-362). Mahwah, NJ: Erlbaum. 
Cook, C., R., Williams, K. R., Guerra, N., Kim, T. \& Sadek, S. (2010). Predictors of Bullying and Victimization in Childhood and Adolescence: A Meta-analytic Investigation. School Psychology Quarterly, 25, 65-83. https://doi.org/10.1037/a0020149

Copeland, W., Wolke, D., Angold, A. \& Costello, J. (2013). Adult psychiatric and suicide outcomes of bullying and being bullied by peers in childhood and adolescence. Journal of the American Medical Association, Psychiatry, 70, 419-426. https://doi. org/10.1001/jamapsychiatry.2013.504

Cornell, D. G. \& Bandyopadhyay, S. (2010). The assessment of bullying. En S. Jimerson, S. Swearer y D. Espelage. (Eds.), Handbook of Bullying in Schools: An International Perspective (pp. 265-276). Nueva York, NY: Routledge.

Craig, W., Harel-Fisch, Y., Fogel-Grinvald, H., Dostaler, S., Hetland, J., Simons-Morton, B., Molcho, M., de Mato, M. G., Overpeck, M., Due, P., \& Pickett, W. (2009). A cross-national profile of bullying and victimization among adolescents in 40 countries. International Journal of Public Health, 54(2), 216-224. https:// doi.org/10.1007/s00038-009-5413-9

Farrington, D. P. \& Ttofi, M. M. (2011). Bullying as a predictor of offending, violence and later life outcomes. Criminal Behaviour and Mental Health, 21, 90-98. https://doi.org/10.1002/ cbm. 801

Fondo para la Infancia de las Naciones Unidad (Unicef) (2011). Clima, conflictos y violencia en la escuela. Buenos Aires: Fondo para la Infancia de las Naciones Unidas.

Gámez-Guadix, M., Villa-George, F. \& Calvete, E. (2014). Psychometric properties of the Cyberbullying Questionnaire (CBQ) among Mexican Adolescents. Violence and Victims, 29, 232-247. https:// doi.org/10.1891/0886-6708.VV-D-12-00163R1

Gonçalves, F. G., Heldt, E., Peixoto, B. N., Rodrigues, G. A., Filipetto, M. \& Guimarães, L. S. P. (2016). Construct validity and reliability of Olweus Bully/Victim Questionnaire-Brazilian 
version. Psicologia: Reflexão e Crítica, 29(1), 27-33. https://doi. org/10.1186/s41155-016-0019-7

Hartung, C., Little, C., S., Allen, E., K. \& Page, M. (2011). A psychometric comparison of two self-report measures of bullying and victimization: Differences by sex and grade. School Mental Health, 3, 44-57. https://doi.org/10.1007/s12310-010-9046-1

Hu, L.T. \& Bentler, P.M. (1999). Cutoff criteria for fit indexes in covariance structure analysis: Conventional criteria versus new alternatives. Structural Equation Modeling, 6(1), 1-55. https:// doi.org/10.1080/10705519909540118

Hymel, S. \& Swearer, S. (2015). Four decades of research on school bullying: An introduction. American Psychologist, 70(4), 293299. https://doi.org/10.1037/a0038928

Jessor, R. \& Jessor, S. (1977). Problem behavior and psychosocial development: A longitudinal study of youth. Nueva York, NY: Academic Press.

Juvonen, J., Graham, S. \& Schuster, M. A. (2003). Bullying among young adolescents: The strong, the weak, and the troubled. Pediatrics, 112(6), 1231-1237. https://doi.org/10.1542/ peds.112.6.1231

Kaltiala-Heino, R. \& Frojd, S. (2011). Correlation between bullying and clinical depression in adolescent patients. Adolescent Health, Medicine and Therapeutics, 2, 37-44. https://doi.org/10.2147/ AHMT.S11554

Kaltiala-Heino, R., Frojd, S. \& Marttunen, M. (2010). Involvement in bullying and depression in a 2-year follow-up in middle adolescence. European Child and Adolescent Psychiatry, 19, 45-55. https://doi.org/10.1007/s00787-009-0039-2

Kaplan, R. M. \& Saccuzzo, D. P. (2006). Pruebas psicológicas: principios, aplicaciones y temas. 6ta ed. Madrid: International Thomson.

Karna, A., Voeten, M., Little, T., Poskiparta, E., Alanen, E. \& Salmivalli, C. (2011). Going to scale: a nonrandomized nationwide trial of the KiVa antibullying program for grade 1-9. Journal 
of Consulting and Clinical Psychology, 79, 796-805. https://doi. org/10.1037/a0025740

Kljakovic, M. \& Hunt, C. (2016). A meta-analysis of predictors of bullying and victimization in adolescence. Journal of Adolescence, 49, 134-145. https://doi.org/10.1016/j.adolescence.2016.03.002

Kovacs, M. (1992). Children's Depression Inventory Manual. North Tonawanda, NY: Multi-Health Systems.

Kowalski, R. M.; Giumetti, G. W., Schroeder, A. N. \& Lattanner, M. R. (2014). Bullying in the digital age: A critical review and meta-analysis of cyberbullying research among youth. Psychological Bulletin, 140(4), 1073-1137. https://doi.org/10.1037/ a0035618

Kowalski, R. M. \& Limber, S. P. (2013). Psychological, physical, and academic correlates of cyberbullying and traditional bullying. Journal of Adolescent Health, 53, 13-20. https://doi. org/10.1016/j.jadohealth.2012.09.018

Kyriakides, L., Kaloyirou, C. \& Lindsay, G. (2006). An analysis of the Revised Olweus Bully/Victim Questionnaire using the Rasch measurement model. British Journal of Educational Psychology, 76(4), 781-801. https://doi.org/10.1348/000709905X53499

LaFontana, K. \& Cillessen, A. (2010). Developmental changes in the priority of perceived status in childhood and adolescence. Social Development, 19(1), 130-147. https://doi. org/10.1111/j.1467-9507.2008.00522.x

Mason, K. L. (2008). Cyberbullying: A preliminary assessment for school personnel. Psychology in the School, 45(4), 323-348. https://doi.org/10.1002/pits.20301

Mehari, K. R., Farrell, A. D. \& Le, A. H. (2014). Cyberbullying among adolescents: Measures in search of a construct. Psychology of Violence, 4(4), 399-415. https://doi.org/10.1037/a0037521

Nansel, T., Craig, W., Overbeck, M., Saluja, G. \& Ruan, W. (2004). Cross-national consistency in the relationship between bullying behaviours and psychosocial adjustment. Paediatric and 
Adolescent Medicine, 158(8), 730-736. https://doi.org/10.1001/ archpedi.158.8.730

Observatorio Argentino de Violencia en las Escuelas. (2008). Violencia en las escuelas: un relevamiento desde la mirada de los alumnos. Buenos Aires, Argentina: Ministerio de Educación.

Olweus, D. (1993). Bullying at school: What we know and what we can do. Cambridge, MA: Blackwell.

Olweus D. (1996). The Revised Olweus Bully/Victim Questionnaire. Bergen, Noruega: HEMIL, Universidad de Bergen.

Olweus, D. (2011). Bullying at school and later criminality: Findings from three Swedish community samples of males. Criminal Behaviour and Mental Health, 11(2), 151-156. https://doi. org/10.1002/cbm.806

Olweus, D. (2012). Invited expert discussion paper: Cyberbullying: An overrated phenomenon? European Journal of Developmental Psychology, 9(5), 520-538. https://doi.org/10.1080/17405629.2 012.682358

Olweus, D. (2013). School bullying: Development and some important challenges. Annual Review of Clinical Psychology, 9, 751-780. https://doi.org/10.1146/annurev-clinpsy-050212-185516

Olweus, D. \& Limber, S. (2018). Some problems with cyberbullying research. Current opinion in psychology, 19, 139-143. https://doi. org/10.1016/j.copsyc.2017.04.012

Patchin, J. \& Hinduja, S. (2015). Measuring cyberbullying: Implications for research. Aggression and Violent Behavior, 23, 69-74. https://doi.org/10.1016/j.avb.2015.05.013

Pellegrini, A. D. \& Long, J. (2010). A longitudinal study of bullying, dominance, and victimization during the transition from primary school through secondary school. British Journal of Developmental Psychology, 20, 259-280. https://doi. org/10.1348/026151002166442

Phillips, V. \& Cornell, D. (2012). Identifying victims of bullying: Use of counselor interviews to confirm peer nominations. Professional 
School Counseling, 15, 123-131. https://doi.org/10.5330/ PSC.n.2012-15.123

Raskauskas, J. \& Stoltz, A. (2007). Involvement in traditional and electronic bullying among adolescents. Developmental Psychology, 43, 564-575. https://doi.org/10.1037/0012-1649.43.3.564

Reijntjes, A., Vermande, M., Goossens, F., Olthof, T., van de Schoot, R, Aleva, L \& van der Meulen, M. (2014). Developmental trajectories of bullying and social dominance in youth. Child Abuse \& Neglect, 37(4), 224-234. https://doi.org/10.1016/j. chiabu.2012.12.004

Resett, S. (2011). Aplicación del cuestionario de agresores/víctimas de Olweus a una muestra de adolescentes argentinos. Revista de Psicología de la Universidad Católica Argentina, 13(7), 27-44.

Roh, B. R., Yoon, Y., Kwon, A., Oh, S., Lee, S. I., Ha, K., ...Hong, H. J. (2015). The structure of co-occurring bullying experiences and associations with suicidal behaviors in Korean adolescents. PloS one, 10, 1-14. https://doi.org/10.1371/journal.pone.0143517

Romera Félix, E., del Rey Alamillo, R. \& Ortega Ruiz, R. (2011). Prevalencia y aspectos diferenciales relativos al género del fenómeno bullying en países pobres. Psicothema, 23(4), 624-629.

Rosenberg, M. (1973). La autoimagen del adolescente y la sociedad. Buenos Aires, Argentina: Paidós.

Rudolph, K. D. (2009). The interpersonal context of adolescent depression. En S. Nolen-Hoeksema y L. Hilt (Eds.), Handbook of adolescent depression (pp. 377-418). Mahwah, NY: Erlbaum.

Rudolph, K. D., Flynn, M. \& Abaied, J. L. (2008). A developmental perspective on interpersonal theories of youth depression. En J. R. Z. Abela y B. L. Hankin (Eds.), Child and adolescent depression: Causes, treatment, and prevention (pp. 79-102). Nueva York, NY: Guilford.

Sheppard, C., Giletta, M. \& Prinstein, M. (2016). Peer victimization trajectories at the adolescent transition: associations among chronic victimization, peer-reported status, and adjustment. 
Journal of Clinical Child \& Adolescent Psychology, 23, 1-10. https://doi.org/10.1080/15374416.2016.1261713

Smith, P. (2016). Bullying: Definition, types, causes, consequences and intervention. Social and Personality Psychology Compass, 10(9), 519-532. https://doi.org/10.1111/spc3.12266

Solberg, M. \& Olweus, D. (2003). Prevalence estimation of school bullying with the Olweus Bully/Victim Questionnaire. Aggressive Behavior, 29, 239-268. https://doi.org/10.1002/ab.10047

Sontag, L. M., Clemans, K., Graber, J. A. \& Lyndon, S. T. (2011). Traditional and cyber aggressors and victims: A comparison of psychosocial characteristics. Journal of Youth and Adolescence, 40, 392- 404. https://doi.org/10.1007/s10964-010-9575-9

Swearer, S. \& Hymel, S. (2015). Understanding the Psychology of Bullying: moving toward a social-ecological diathesis stress model. American Psychologist, 70, 344-353. https://doi.org/10.1037/ a0038929

Troop-Gordon, W. (2017). Peer victimization in adolescence: The nature, progression, and consequences of being bullied within a developmental context. Journal of Adolescence, 55, 116-128. https://doi.org/10.1016/j.adolescence.2016.12.012

Ttofi, M. M., Farrington, D. P. \& Lösel, F. (2011). Do the victims of school bullies tend to become depressed later in life? A systematic review and meta-analysis of longitudinal studies. Journal of Aggression, Conflict and Peace Research, 3, 63-73. https://doi. org/10.1108/17596591111132873

Yeager, D. S., Fong, C. J., Lee, H. Y. \& Espelage, D. L. (2015). Declines in efficacy of anti-bullying programs among older adolescents: Theory and a three-level meta-analysis. Journal of Applied Developmental Psychology, 37, 36-51. https://doi.org/10.1016/j. appdev.2014.11.005

Recibido: 17 de abril, 2017 Revisado: 16 de octubre, 2017 Aceptado: 30 de marzo, 2018 